\title{
Encyclopedia of Biometrics
}



Stan Z. Li • Anil K. Jain

Editors

\section{Encyclopedia of Biometrics}

Second Edition

With 823 Figures and 102 Tables

Springer Reference 


\section{Editors}

Stan Z. Li

Center for Biometrics and Security

Research \& National Laboratory

of Pattern Recognition

Institute of Automation

Chinese Academy of Sciences

Beijing, China

\author{
Anil K. Jain \\ Department of Computer Science \\ and Engineering \\ Michigan State University \\ East Lansing, MI, USA
}

ISBN 978-1-4899-7487-7 ISBN 978-1-4899-7488-4 (eBook)

ISBN 978-1-4899-7489-1 (print and electronic bundle)

DOI 10.1007/978-1-4899-7488-4

Library of Congress Control Number: 2015936276

Springer New York Heidelberg Dordrecht London

(C) Springer Science+Business Media New York 2009, 2015

This work is subject to copyright. All rights are reserved by the Publisher, whether the whole or part of the material is concerned, specifically the rights of translation, reprinting, reuse of illustrations, recitation, broadcasting, reproduction on microfilms or in any other physical way, and transmission or information storage and retrieval, electronic adaptation, computer software, or by similar or dissimilar methodology now known or hereafter developed.

The use of general descriptive names, registered names, trademarks, service marks, etc. in this publication does not imply, even in the absence of a specific statement, that such names are exempt from the relevant protective laws and regulations and therefore free for general use.

The publisher, the authors and the editors are safe to assume that the advice and information in this book are believed to be true and accurate at the date of publication. Neither the publisher nor the authors or the editors give a warranty, express or implied, with respect to the material contained herein or for any errors or omissions that may have been made.

Printed on acid-free paper

Springer Science+Business Media LLC New York is part of Springer Science+Business Media (www.springer.com) 


\section{Preface to the Second Edition}

The Encyclopedia of Biometrics provides a comprehensive reference to concepts, definitions, technologies, challenges, and trends in the field of biometrics. The volume covers all important aspects in biometrics research, development, and applications, including sensors and devices, methods and algorithms, sample quality, system design and implementation, databases, performance testing, security, antispoofing, and standards.

The second edition consists of over 250 in-depth articles contributed by about 300 leading biometric experts around the world. Major updates in the first edition, published in 2009, are as follows:

1. Addition of a new section on biometric spoofing attacks and antispoofing methods

2. Update of the biometric standards section

3. Entries on new biometric modalities, such as EEG biometrics, pointerbased recognition, and periocular recognition

4. Addition of entries on newly encountered problems, such as effect of plastic surgery on face recognition, remote (at a distance) face recognition, and mixing fingerprints for security

5. New algorithms for age estimation, gender classification, and score normalization

The focus of the Encyclopedia is on up-to-date, yet comprehensive, information in an easy-to-use format that is accessible to researchers and scientists, students, system designers, engineers, practitioners, and policymakers working in the broad field of biometrics. It is available as a print edition as well as a fully searchable e-version with extensive cross-referencing and updates as new trends and terms arise in the rapidly growing field of biometrics.

\section{Key Features at a Glance}

- Serves as a single point of entry for in-depth and current information on biometrics

- Covers major biometric modalities of face, fingerprint, iris, vein, voice, and hand as well as other modalities of interest in both biometrics and forensics (ear, gait, skin, tooth, odor, skull, DNA)

- Follows an A-Z format that allows intuitive and easy-to-use access

- Consists of cross-referenced entries 
- Has a distinguished editorial board from across the scientific and engineering disciplines related to biometrics

- Provides a balanced coverage of research and development in biometrics

\section{Acknowledgments}

We are grateful to a number of individuals who have assisted us in the production of the second edition of the Encyclopedia of Biometrics. First of all, our deep gratitude to Shengcai Liao and Zhen Lei for their proofreading and ensuring that all the authors provided their contributions on time. Our stellar team of area editors has done excellent work in writing, inviting, and reviewing contributions from the experts in their respective topics. Our sincere thanks to Andy Adler, Joseph Campbell, Raffaele Cappelli, Christophe Champod, Stephen Elliott, Julian Fierrez, Christophe Fondeur, Carmen GarcíaMateo, Josef Kittler, Hale Kim, Ajay Kumar, Davide Maltoni, Aleix Martinez, Mark Nixon, Geppy Parziale, Fernando Podio, Salil Prabhakar, Arun Ross, Marios Savvides, Yoichi Seto, Colin Soutar, Wei-Yun Yau, Pong C. Yuen, David Zhang, Sébastien Marcel, and John Daugman. This book would not have been possible without the support of the numerous authors who provided their contributions. Special thanks are due to the Springer staff for their advice, support, and, most importantly, patience. Neha Thapa and Sunaina Dadhwal deserve special mention for gently shepherding us throughout the book preparation and production process.

9th March 2015

Stan Z. Li

Beijing, China

Anil K. Jain

MI, USA 


\section{Preface to the First Edition}

The Encyclopedia of Biometrics provides a comprehensive reference to concepts, technologies, issues, and trends in the field of biometrics. The volume covers all important aspects-research, development, and applications, including biometric sensors and devices, methods and algorithms, sample quality, system design and implementation, databases, performance testing, information security, and standardization. Leading experts around the world contributed to this collection of over 200 in-depth essays accompanied by more than 600 definitional entries.

The focus of the encyclopedia is on immediate, yet comprehensive, information in an easy-to-use format that is accessible to researchers and scientists, system designers, engineers, programmers, students, practitioners, and government agents working in the broad field of biometrics. It is available as a print edition as well as a fully searchable version with extensive crossreferencing and updates as new trends and terms arise.

\section{Key Features at a Glance}

- Serves as an immediate point of entry into the field for in-depth research

- Covers biometrics of the face, fingerprint, iris, vein, voice, hand, ear, gait, skin, tongue, tooth, odor, skull, and DNA

- Follows an A-Z format that allows intuitive and easy-to-use access

- Consists of cross-referenced entries

- Has an internationally renowned editorial board from across the scientific and engineering disciplines and geographies

- Provides balanced coverage

\section{Acknowledgments}

I am grateful to all the people who have played a part in the production of this encyclopedia. First of all, my deep gratitude to Anil K. Jain, Editorial Advisor, for sharing his knowledge, expertise, and important advice and suggestions for this encyclopedia. Our stellar team of area editors has done excellent work in writing, inviting, and reviewing contributions from many leaders of the field. My sincere thanks to Andy Adler, Joseph Campbell, Raffaele Cappelli, Christophe Champod, Stephen Elliott, Julian Fierrez, 
Jean-Christophe Fondeur, Carmen García-Mateo, Josef Kittler, Hale Kim, Ajay Kumar, Davide Maltoni, Aleix Martinez, Mark Nixon, Geppy Parziale, Fernando Podio, Salil Prabhakar, Arun Ross, Marios Savvides, Yoichi Seto, Colin Soutar, Wei-Yun Yau, Pong C. Yuen, and David Zhang. I would like to thank these area editors for their help in creating this book and the numerous authors for their individual contributions. Special thanks are due to people at Springer for their enthusiasm, advice, and support. Jennifer Evans, Susan Lagerstrom-Fife, Michaela Bilic, Tina Shelton, and Anil Chandy have played key roles at different times in the development of this book.

Beijing, China

Stan Z. Li 


\section{About the Editors}

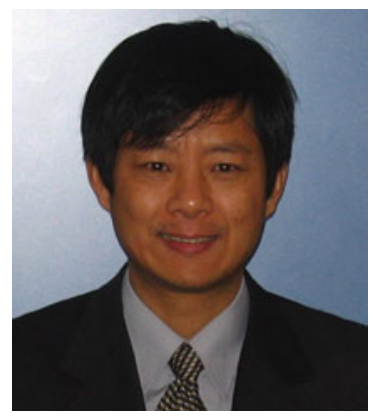

Stan Z. Li Center for Biometrics and Security Research, National Laboratory of Pattern Recognition, Institute of Automation, Chinese Academy of Sciences, Beijing, China.

An IEEE Fellow, Stan Z. Li received his B.Eng. from Hunan University, China, M.Eng. from National University of Defense Technology, China, and $\mathrm{Ph}$.D. degree from Surrey University, UK. He is currently a professor at the National Laboratory of Pattern Recognition and the director of the Center for Biometrics and Security Research (CBSR), Institute of Automation (CASIA), and the director of the Center for Visual Internet of Things Research (VIOT), Chinese Academy of Sciences. He worked at Microsoft Research Asia as a researcher from 2000 to 2004. Prior to that, he was an associate professor at Nanyang Technological University, Singapore. He was elevated to for his contributions to the fields of face recognition, pattern recognition, and computer vision.

His research interest includes pattern recognition and machine learning, image and vision processing, face recognition, and video analytics. He has published over 300 papers in international journals and conferences and authored and edited eight books, among which Markov Random Field Models in Image Analysis (Springer, 1st edition 1995, 2nd edition 2001, 3rd edition 2009) has been cited more than 2000 times (by Google Scholar). Other works include Handbook of Face Recognition (Springer, 1st edition 2005, 2nd edition 2011) and Encyclopedia of Biometrics (Springer Reference Work, 1st edition 2010, 2nd edition 2014). He served as an associate editor of IEEE 
Transactions on Pattern Analysis and Machine Intelligence and a cochair of the International Conference on Biometrics 2007 and 2009 and has been involved in organizing other international conferences and workshops in the fields of his research interest.

$\mathrm{Stan} \mathrm{Z}$. $\mathrm{Li}$ is an expert in face recognition, biometrics, and intelligent video surveillance. The EyeCU face recognition system he developed at Microsoft Research Asia was demonstrated by Bill Gates on a CNN interview. He has been leading several national and international projects in biometrics and intelligent video surveillance. The AuthenMetric face recognition system and intelligent video surveillance system have been deployed in several national projects, including Beijing 2008 Olympic Games, Shanghai 2010 World Expo, and immigration control at China borders. He is a cochair of SAC/TC100/SC2 for biometrics standardization in China and delivered a plenary speech on Biometrics in China at ISO/IEC JTC1/SC37 on behalf of the China National Body.

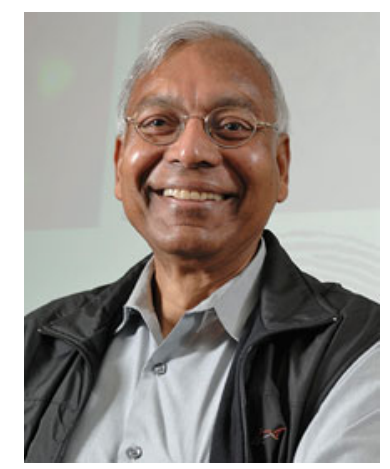

Anil K. Jain Department of Computer Science and Engineering, Michigan State University, East Lansing, MI, USA

Anil K. Jain is a University Distinguished Professor in the Department of Computer Science and Engineering at Michigan State University. He received his B.Tech. degree from the Indian Institute of Technology, Kanpur (1969), and M.S. and Ph.D. degrees from The Ohio State University in 1970 and 1973, respectively. His research interests include pattern recognition, computer vision, and biometric recognition. His articles on biometrics have appeared in Scientific American, Nature, IEEE Spectrum, Comm. ACM, IEEE Computer, Proc. IEEE, Encarta, Scholarpedia, and MIT Technology Review.

$\mathrm{He}$ has received Guggenheim fellowship, Humboldt Research Award, Fulbright fellowship, IEEE Computer Society Technical Achievement Award, IEEE W. Wallace McDowell Award, IAPR King-Sun Fu Prize, IEEE ICDM Research Contribution Award, and the MSU Withrow Teaching Excellence Award for contributions to pattern recognition and biometrics. $\mathrm{He}$ also received the best paper awards from the IEEE Transactions on Neural Networks (1996) and the Pattern Recognition journal (1987, 1991, and 2005). He served as the Editor-in-Chief of the IEEE Transactions on Pattern Analysis and Machine Intelligence (1991-1994). He is a Fellow of the ACM, IEEE, 
AAAS, IAPR, and SPIE. He has been listed among the "18 Indian Minds Who Are Doing Cutting Edge Work" in the fields of science and technology and felicitated with the 2014 Innovator of the Year Award at MSU.

Anil K. Jain has been assigned six US patents on fingerprint matching and two Korean patents on surveillance. He is the author of several books, including Introduction to Biometrics (2011), Handbook of Biometrics (2007), Handbook of Multibiometrics (2006), Handbook of Face Recognition (first edition 2005, second edition 2011), Handbook of Fingerprint Recognition (first edition 2003, second edition 2009) (received the PSP award from the Association of American Publishers), Markov Random Fields: Theory and Applications (1993), and Algorithms for Clustering Data (1988). ISI has designated him as a highly cited researcher (Scholar). According to CiteSeer, his book, Algorithms for Clustering Data is ranked \# 75 in the Most Cited Articles in Computer Science (over all times) and his paper "Data Clustering: A Review" (ACM Computing Surveys, 1999) is consistently ranked in the Top 10 Most Popular Magazine and Computing Survey Articles Downloaded.

He served as a member of the National Academies panels on Information Technology, Whither Biometrics, and Improvised Explosive Devices (IED). $\mathrm{He}$ also served as a member of the Defense Science Board. He currently serves as a member of the Forensic Science Standards Board. 



\section{Assistant Editors}

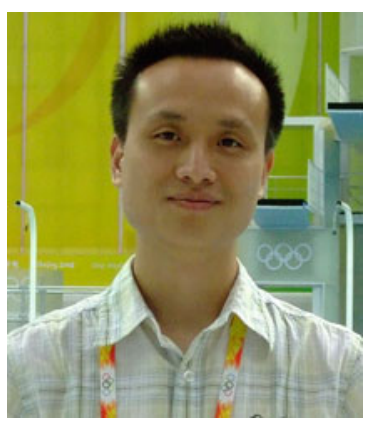

\section{Shengcai Liao}

Center for Biometrics and

Security Research (CBSR),

National Laboratory of Pattern

Recognition (NLPR),

Institute of Automation,

Chinese Academy of Sciences (CASIA),

Beijing, China

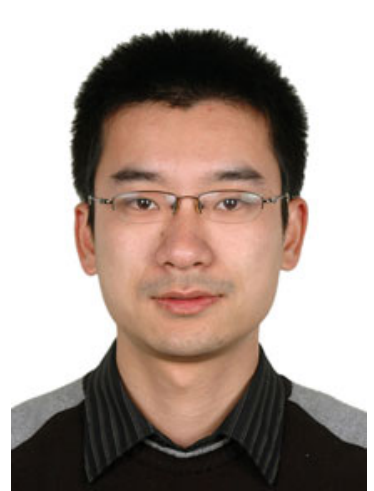

\section{Zhen Lei}

Center for Biometrics and

Security Research,

National Laboratory of Pattern Recognition,

Institute of Automation,

Chinese Academy of Sciences,

Beijing, China 



\title{
Section Editors
}

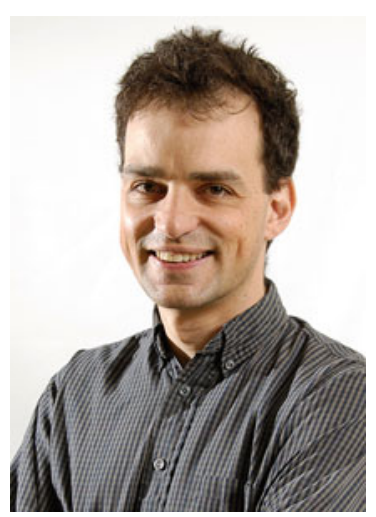

\author{
Andy Adler \\ Department of Systems and \\ Computer Engineering, \\ Carleton University, \\ Ottawa, ON, Canada
}

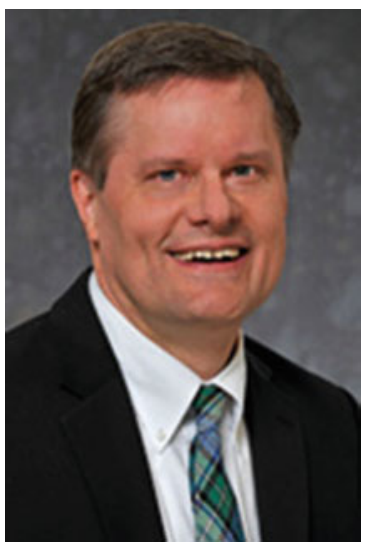

Joseph P. Campbell

Lincoln Laboratory,

Massachusetts Institute of Technology, Human Language Technology Group, Lexington, MA, USA

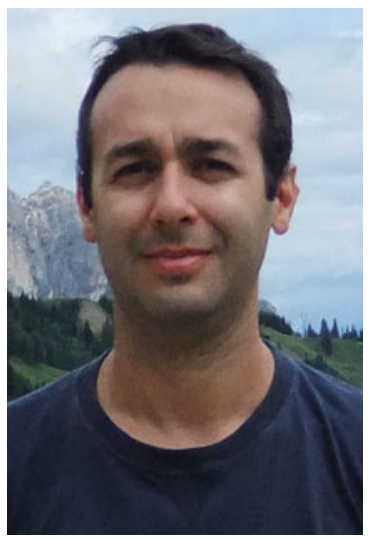

\section{Raffaele Cappelli}

Department of Computer Science and Engineering (DISI),

University of Bologna, Cesena (FC), Italy 

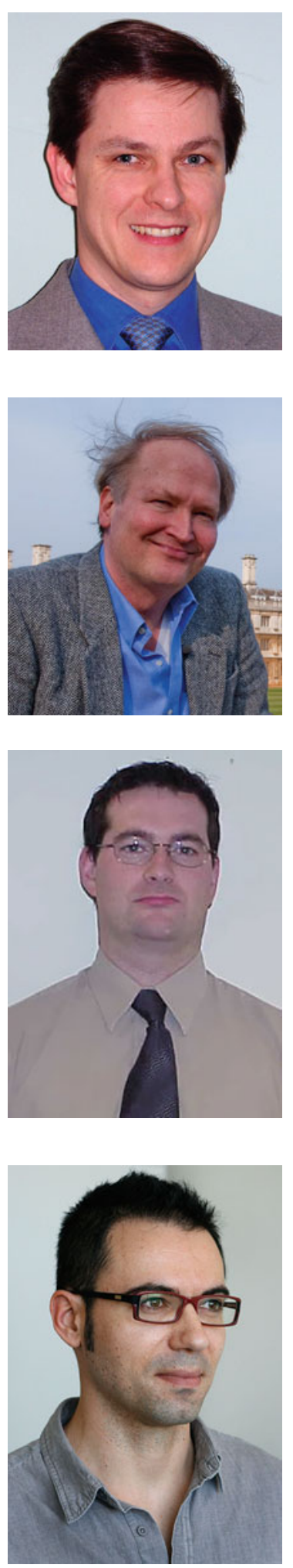

\section{Christophe Champod}

School of Criminal Justice -

Forensic Science,

University of Lausanne,

Lausanne, Switzerland

\section{John Daugman}

University of Cambridge,

Cambridge, UK

\section{Stephen John Elliott}

International Center for Biometric Research,

Purdue University,

West Lafayette,

IN, USA

\section{Julian Fierrez}

Universidad Autonoma de Madrid,

Madrid, Spain 


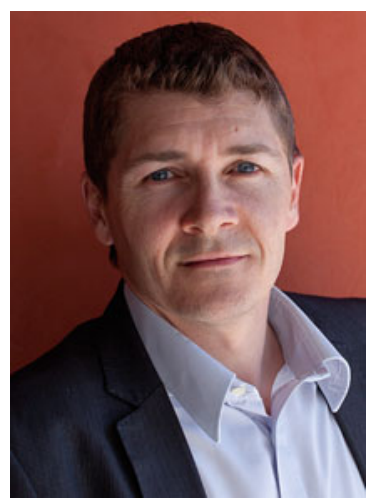

\section{Jean-Christophe Fondeur}

Research \& Technology,

Morpho, Paris, France

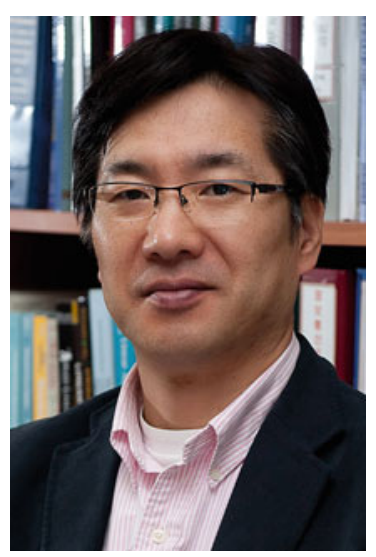

\section{Hakil Kim}

Department of Information and

Communication Engineering,

Inha University,

Incheon, Korea

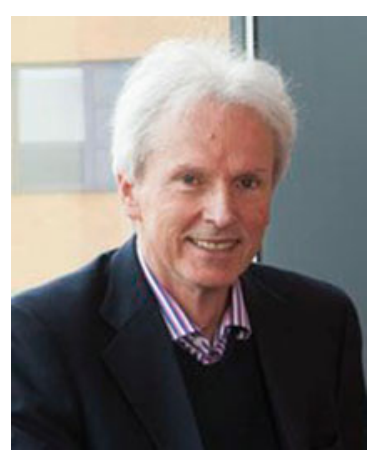

\section{Josef Kittler}

Department of Electronic Engineering, Centre for Vision,

Speech and Signal Processing, University of Surrey,

Guildford, UK

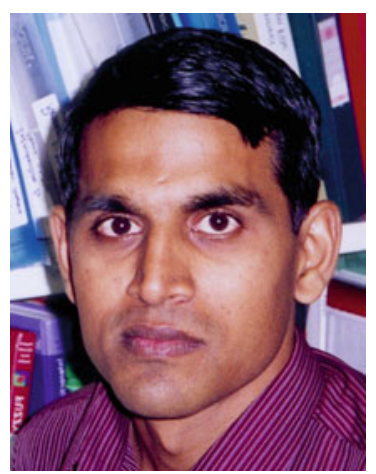

\section{Ajay Kumar}

The Hong Kong Polytechnic University, Hung Hom, Kowloon,

Hong Kong 


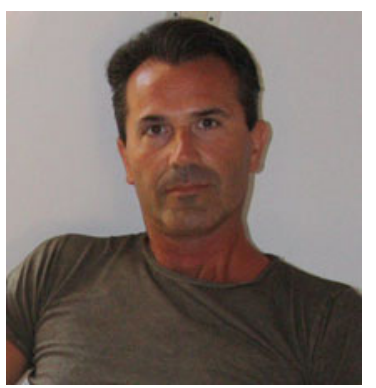

\section{Davide Maltoni}

Department of Computer Science

and Engineering,

University of Bologna,

Bologna, Cesena, Italy

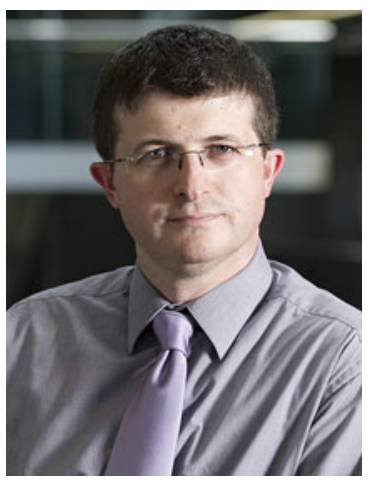

\section{Sébastien Marcel}

Idiap Research Institute,

Martigny, Switzerland

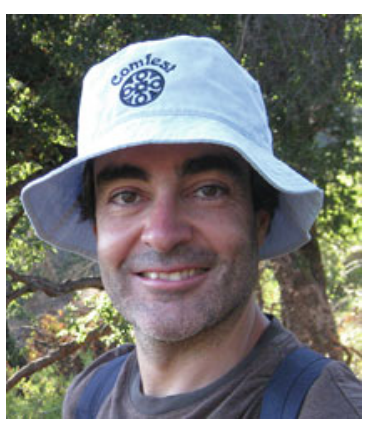

\section{Aleix M. Martinez}

Department of Electrical and

Computer Engineering,

Ohio State University,

Columbus, OH, USA

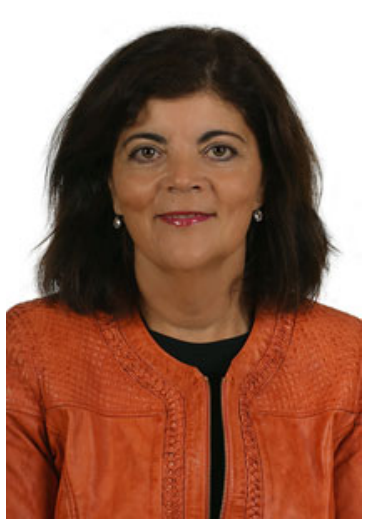

\section{Carmen García Mateo}

Atlantic Research Center for

Information and Communication

Technologies,

University of Vigo,

Pontevedra, Spain 


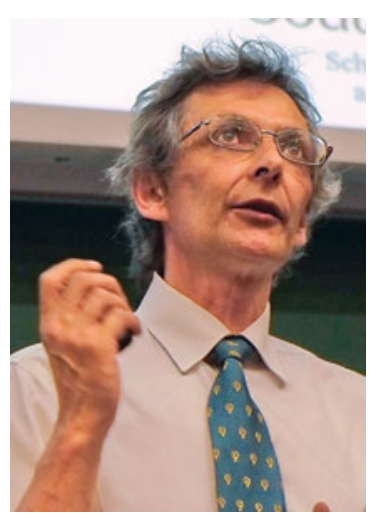

\section{Mark Nixon}

School of Electronics and

Computer Science,

University of Southampton,

Southampton, UK

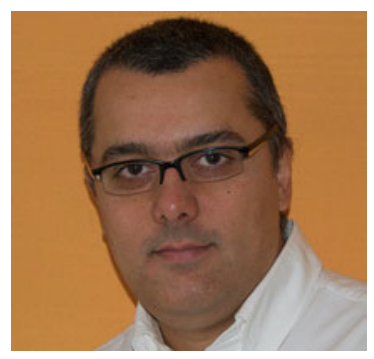

\section{Geppy Parziale}

INVASIVECODE INC.,

San Francisco,

CA, USA

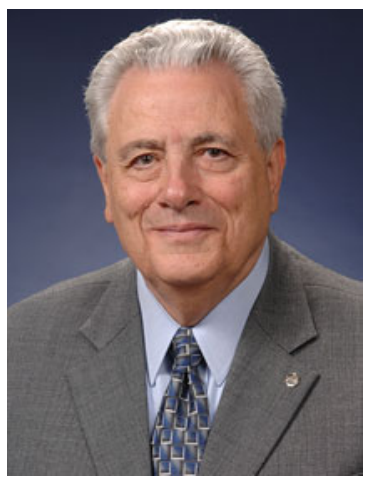

\section{Fernando Podio}

Computer Security Division,

National Institute of Standards and Technology,

Gaithersburg, MD, USA

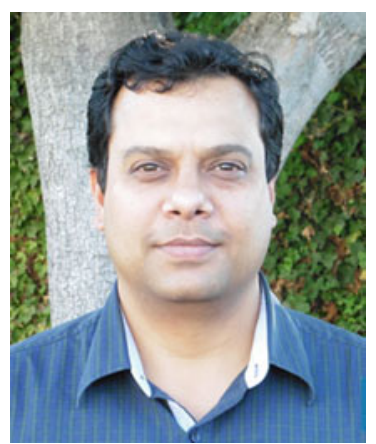

\section{Salil Prabhakar}

Delta ID Inc.,

Newark,

CA, USA 


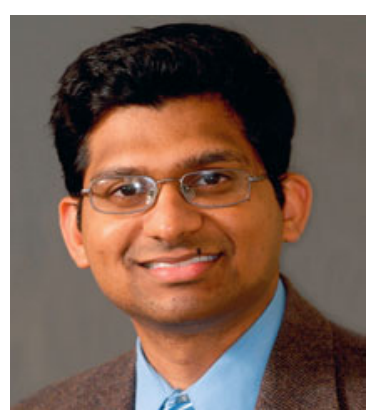

\section{Arun Ross}

Michigan State University,

East Lansing,

MI, USA

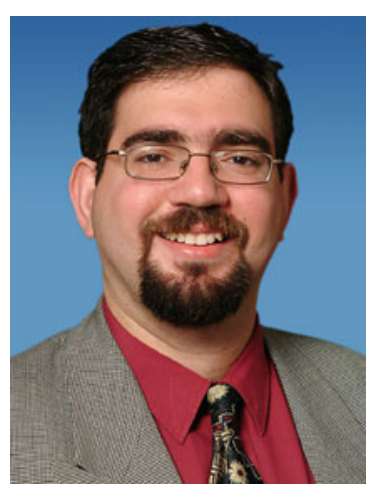

\section{Marios Savvides}

CMU CyLab Biometrics Center,

Pittsburgh,

PA, USA

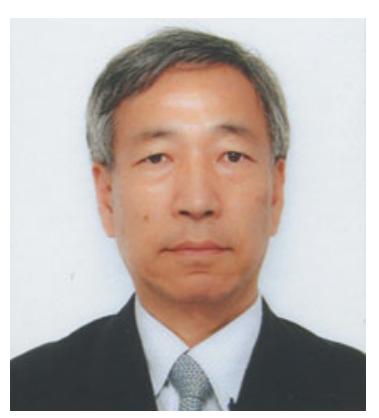

\section{Yoichi Seto}

Advanced Institute of

Industrial Technology,

Tokyo Metropolitan University,

Tokyo, Japan

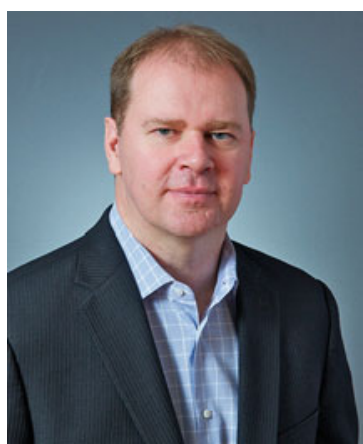

\section{Colin Soutar}

Cyber Risk Services,

Deloitte and Touche LLP,

Arlington,

VA, USA 


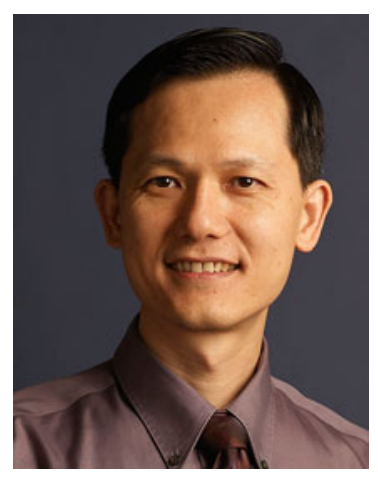

\section{Wei-Yun Yau}

Institute for Infocomm Research,

Singapore

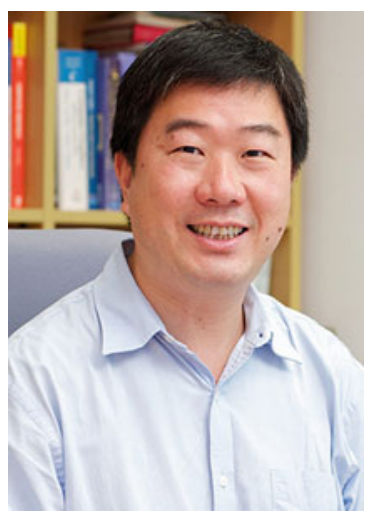

\section{Pong C. Yuen}

Department of Computer Science, Hong Kong Baptist University,

Kowloon, Hong Kong

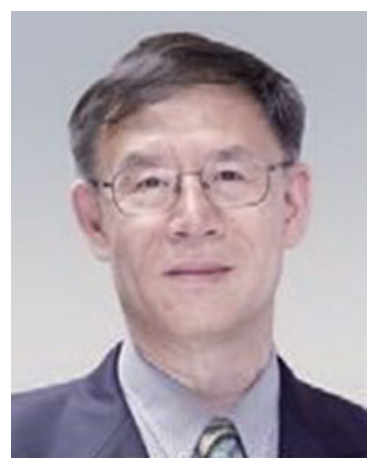

\section{David Zhang}

Biometrics Research Centre,

Department of Computing,

The Hong Kong Polytechnic University,

Hung Hom, Kowloon,

Hong Kong 



\section{Contributors}

Mongi Abidi The University of Tennessee, Knoxville, TN, USA

Mathias M. Adankon ML-Consulting, Laval, QC, Canada

Andy Adler Department of Systems and Computer Engineering, Carleton University, Ottawa, ON, Canada

Gaurav Aggarwal University of Maryland, College Park, MD, USA

Jonathan R. Agre IDA Information Systems and Technology Division, Institute for Defense Analyses, Alexandria, VA, USA

Thomas Albrecht Computer Science Department, University of Basel, Basel, Switzerland

Federico Alegre EURECOM, Biot, France

Petar S. Aleksic Google Inc., New York, NY, USA

Nigel M. Allinson Department of Electronic and Electrical Engineering, University of Sheffield, Sheffield, UK

Fernando Alonso-Fernandez Intelligent Systems Lab (IS-Lab/CAISR), Halmstad University, Halmstad, Sweden

André Anjos Idiap Research Institute, Martigny, Switzerland

Takahiro Aoki Secure Computing Laboratory, Fujitsu Laboratories Ltd., Kawasaki, Japan

Sean Banerjee Lane Department of Computer Science and Electrical Engineering, West Virginia University, Morgantown, WV, USA

Nick Bartlow Noblis, Inc., Falls Church, VA, USA

Alex Bazin Fujitsu Services, London, UK

George Bebis Department of Computer Science and Engineering, University of Nevada, Reno, NV, USA

Robert Bell Biometrics Alliance, Severna Park, MD, USA

Herman Bergman Oakland police department criminalistics division, Oakland, CA, USA 
Bir Bhanu Center for Research in Intelligent Systems, University of California, Riverside, CA, USA

Samarth Bharadwaj Image Analysis and Biometrics Lab, IIIT-Delhi, New Delhi, India

Himanshu Sharad Bhatt Image Analysis and Biometrics Lab, IIIT-Delhi, New Delhi, India

Battista Biggio Department of Electrical and Electronic Engineering, University of Cagliari, Cagliari, Italy

Josef Bigun Embedded Intelligent Systems Center, Halmstad University, IDE, Halmstad, Sweden

Vance Bjorn DigitalPersona Inc., Redwood City, CA, USA

Ramón Blanco-Gonzalo Departamento de Tecnologia Electronica, University Carlos III of Madrid, Leganes, Madrid, Spain

Electrical and Electronic Department, Universidad Publica de Navarra, Pamplona, Navarra, Spain

Jean-François Bonastre CERI, University of Avignon, Avignon, France

Jeffrey E. Boyd University of Calgary, Calgary, AB, Canada

Michael Brockly Department of Technology, Leadership and Innovation, Purdue University, West Lafayette, IN, USA

Michael C. Bromby Glasgow Caledonian University, Glasgow, UK

Randy Broussard Applied Physics Laboratory, The John Hopkins University, Annapolis, MD, USA

Kelvin Bryant North Carolina A\&T State University, Greensboro, $\mathrm{NC}$, USA

Douglas J. Buettner The Aerospace Corporation, El Segundo, CA, USA

Anne M. Burrows Duquesne University, Pittsburgh, PA, USA

Christoph Busch Fraunhofer-IGD, Darmstadt, Germany

Gjøvik University College, Gjøvik, Norway

Thomas A. Busey Psychological and Brain Sciences, Program in Cognitive Science, Indiana University, Bloomington, IN, USA

James L. Cambier Crossmatch Technologies, RCA Blvd, FL, USA

John W.M. Campbell Bion Biometrics Inc., Nepean, ON, Canada

Patrizio Campisi Section of Applied Electronics, Department of Engineering, Roma Tre University, Rome, Italy

Greg Cannon Cross Match Technologies, Palm Beach Gardens, FL, USA 
Kai Cao Center for Biometrics and Security Research \& The Key Laboratory of Complex System and Intelligence Science Chinese Academy of Sciences, Institute of Automation Zhingguancun Donglu, Beijing, China

Raffaele Cappelli Department of Computer Science and Engineering (DISI), University of Bologna, Cesena (FC), Italy

Carlos D. Castillo Department of Computer Science, University of Maryland, College Park, MD, USA

Ann Cavoukian Office of the Information and Privacy Commissioner, Toronto, ON, Canada

Christophe Champod School of Criminal Justice - Forensic Science, University of Lausanne, Lausanne, Switzerland

Rama Chellappa University of Maryland, College Park, MD, USA

Hong Chen Departments of Computer Science and Engineering, Michigan State University, East Lansing, MI, USA

Hui Chen Center for Research in Intelligent Systems, University of California, Riverside, CA, USA

Xilin Chen Institute of Computing Technology, Chinese Academy of Sciences, Beijing, China

Yi Chen Department of Computer Science and Engineering, Michigan State University, MI, USA

Mohamed Cheriet University of Quebec, ETS, Montreal, Canada

Girija Chetty Faculty of ESTeM, University of Canberra, Bruce, ACT, Australia

Ivana Chingovska Idiap Research Institute, Martigny, Switzerland

Alex Hwansoo Choi Techsphere Co., Ltd., Seoul, South Korea

Seungjin Choi Department of Computer Science, Pohang University of Science and Technology, Pohang, South Korea

Michal Choras Image Processing Group, Institute of Telecommunications, University of Technology and Life Sciences, Bydgoszcz, Poland

Stephen Clarke Jebel Consultant Group, Belconnen, ACT, Australia

Jeffrey F. Cohn University of Pittsburgh, Pittsburgh, PA, USA

Alistair Conkie AT\&T Labs Research, Florham Park, NJ, USA

R. Coquoz Institut de police scientifique, Ecole des sciences criminelles, Lausanne, Switzerland

Bojan Cukic Lane Department of Computer Science and Electrical Engineering, West Virginia University, Morgantown, WV, USA 
Allison M. Curran Department of Chemistry and Biochemistry, International Forensic Research Institute, Florida International University, Miami, FL, USA

Qionghai Dai Department of Automation, Tsinghua University, Beijing, China

Sarat C. Dass Universiti Teknologi PETRONAS, Perak, Malaysia

John Daugman University of Cambridge, Cambridge, UK

James W. Davis Ohio State University, Columbus, OH, USA

David Day International Biometric Group, New York, NY, USA

Farzin Deravi University of Kent, Canterbury, Kent, UK

Laura Docio-Fernandez Department of Signal Theory and Communications, University of Vigo, Vigo, Spain

Cathryn Downing Computer Laboratory, Cambridge University, Cambridge, UK

Gerry Vernon Dozier North Carolina A\&T State University, Greensboro, NC, USA

Andrzej Drygajlo Swiss Federal Institute of Technology Lausanne (EPFL), Lausanne, Switzerland

Nicolae Duta Microsoft, Cambridge, MA, USA

Ahmed Elgammal Department of Computer Science, Rutgers University, Piscataway, NJ, USA

Stephen John Elliott International Center for Biometric Research, Purdue University, West Lafayette, IN, USA

Nicholas Evans EURECOM, Biot, France

Kelly Smith Faddis West Virginia University, Morgantown, WV, USA

Belen Fernandez-Saavedra Science Park, ID Testing Lab, Carlos III University of Madrid, Leganes, Madrid, Spain

Julian Fierrez Universidad Autonoma de Madrid, Madrid, Spain

Jean-Christophe Fondeur Research \& Technology, Morpho, Paris, France

Marc Friedman Retica Systems, Inc., Waltham, MA, USA

Pascal Fua EPFL, IC-CVLab, Lausanne, Switzerland

Giorgio Fumera Department of Electrical and Electronic Engineering, University of Cagliari, Cagliari, Italy

Kenneth G. Furton Department of Chemistry and Biochemistry, International Forensic Research Institute, Florida International University, Miami, FL, USA 
Javier Galbally Institute for the Protection and Security of the Citizen, Joint Research Centre European Commission, Ispra (VA), Italy

Wen Gao Peking University, Beijing, China

Lu Gao Omni Vision CDM Optics, Boulder, CO, USA

Carmen García Mateo Atlantic Research Center for Information and Communication Technologies, University of Vigo, Pontevedra, Spain

Sonia Garcia-Salicetti Institut Mines-Télécom/Télécom SudParis, CNRS UMR 5157 SAMOVAR, Evry, France

Xin Geng School of Computer Science and Engineering, Southeast University, Nanjing, China

Shaogang Gong Queen Mary University of London, London, UK

Joaquín González-Rodríguez ATVS - Biometric Recognition Group, Escuela Politecnica Superior, Universidad Autonoma de Madrid, Madrid, Spain

Javier Gonzalez-Dominguez ATVS - Biometric Recognition Group, Escuela Politecnica Superior, Universidad Autonoma de Madrid, Madrid, Spain

Dmitry O. Gorodnichy Laboratory and Scientific Services Directorate, Canada Border Services Agency, Ottawa, ON, Canada

Joseph van der Gracht Holospex Inc., Columbia, MD, USA

Patrick Grother National Institute of Standards and Technology, Gaithersburg, MD, USA

Leon Gu Carnegie Mellon University, Pittsburgh, PA, USA

Richard Guest School of Engineering and Digital Arts, University of Kent, Canterbury, Kent, UK

Guodong Guo Lane Department of Computer Science and Electrical Engineering, West Virginia University, Morgantown, WV, USA

David L. Guyton The Wilmer Ophthalmological Institute, The Johns Hopkins University School of Medicine, Baltimore, MD, USA

Abdenour Hadid Machine Vision Group, Department of Electrical and Information Engineering, University of Oulu, Oulu, Finland

Souichi Hama Secure Computing Laboratory, Fujitsu Laboratories Ltd., Kawasaki, Japan

Onur C. Hamsici The Ohio State University, Columbus, OH, USA

Byoung-Jin Han Electronics and Telecommunications Research Institute, Daejeon, Korea

Seiichiro Hangai Department of Electrical Engineering, Tokyo University of Science, Tokyo, Japan 
Masanori Hara NEC Corporation, Tokyo, Japan

Dominique Harrington Tygart Technology Inc., Fairmont, MV, USA

Jean Hennebert Department of Informatics, University of Fribourg, Fribourg, Switzerland

Institute of Business Information Systems HES-SO Valais, TechnoArk, Sierre, Switzerland

Olaf Henniger Fraunhofer Institute for Computer Graphics Research IGD, Darmstadt, Germany

Javier Hernando Technical University of Catalonia, Barcelona, Spain

Fred Herr Identification Technology Partners, Inc., Gaithersburg, MD, USA

R. Austin Hicklin Noblis, Falls Church, VA, USA

T. Hicks Institut de police scientifique, Ecole des sciences criminelles, Lausanne, Switzerland

Peter T. Higgins Higgins \& Associates, International (HAI), Washington DC, USA

Hisao Ogata Mitsutoshi Himaga Hitachi-Omron Terminal Solutions, Corp., Owari-asahi City, Aichi, Japan

Tom Hopper Criminal Justice Information Services Division, FBI, Washington, DC, USA

Nesma Houmani SIGMA Laboratory, ESPCI Paris Tech, Paris, France

Wen-Hsing Hsu National Tsing Hua University, Taiwan, Republic of China

Dunxu Hu Department of Computer Science, Rutgers University, Piscataway, NJ, USA

David J. Hurley School of Electronics and Computer Science, University of Southampton, Southampton, UK

Kristina Irsch The Wilmer Ophthalmological Institute, The Johns Hopkins University School of Medicine, Baltimore, MD, USA

Robert Ives Applied Physics Laboratory, The John Hopkins University, Annapolis, MD, USA

David W. Jacobs Department of Computer Science, University of Maryland, College Park, MD, USA

Anil K. Jain Department of Computer Science and Engineering, Michigan State University, East Lansing, MI, USA

Jihyeon Jang Inha University, Incheon, Korea

Kui Jia Shenzhen Institute of Advanced Integration Technology, CAS/CUHK, Shenzhen, People's Republic of China

Xudong Jiang Nanyang Technological University, Singapore 
Zhong Jin School of Computer Science and Technology, Nanjing University of Science and Technology, Nanjing, People's Republic of China

Bethany S. Jurs Department of Psychology, Transylvania University, Lexington, NY, USA

Ioannis A. Kakadiaris Department of Computer Science, ECE and Biomedical Engineering, University of Houston, Houston, TX, USA

Nathan Kalka West Virginia University, Morgantown, WV, USA

Takeo Kanade Carnegie Mellon University, Pittsburgh, PA, USA

Vivek Kanhangad Biometrics Research Centre, Department of Computing, The Hong Kong Polytechnic University, Hung Hom, Kowloon, Hong Kong

Christiane Kaplan SOFTPRO GmbH, Böblingen, Germany

Mark Keck Ohio State University, Columbus, OH, USA

Robert B. Kennedy Royal Canadian Mounted Police, Ottawa, ON, Canada

Lauren R. Kennell Applied Physics Laboratory, The John Hopkins University, Annapolis, MD, USA

Hale Kim Inha University, Incheon, Korea

Jong Kyoung Kim Department of Computer Science, Pohang University of Science and Technology, Pohang, South Korea

Kye-Hyeon Kim Department of Computer Science, Pohang University of Science and Technology, Pohang, South Korea

Tomi Kinnunen University of Eastern Finland (UEF), Joensuu, Finland

Naohisa Komatsu Waseda University, Shinjuku-ku, Tokyo, Japan

Eric P. Kukula Department of Industrial Technology, Purdue University, West Lafayette, IN, USA

Ajay Kumar The Hong Kong Polytechnic University, Hung Hom, Kowloon, Hong Kong

Amioy Kumar Biometrics Research Laboratory, Department of Electrical Engineering, Indian Institute of Technology Delhi, New Delhi, India

B.V.K. Vijaya Kumar Department of Electrical and Computer Engineering, Carnegie Mellon University, Pittsburgh, PA, USA

Young-Bin Kwon School of Computer Science and Engineering, Chung-Ang University, Seoul, Korea

Jian-Huang Lai School of Information Science and Technology, Sun Yat-sen University, Guangzhou, Guangdong, People's Republic of China

Kin-Man Lam Department of Electronic and Information Engineering, The Hong Kong Polytechnic University, Hong Kong, China 
Peter K. Larsen Laboratory of Biological Anthropology, Institute of Forensic Medicine, University of Copenhagen, Copenhagen, Denmark

Richard T. Lazarick Computer Sciences Corporation Identity Lab, Ewing, NJ, USA

Victor Minchih Lee International Biometric Group, New York, NY, USA

Yooyoung Lee Dakota-consulting Inc., Contractor at National Institute of Standards and Technology (NIST), Gaithersburg, MD, USA

Graham Leedham School of Information and Communication Technology, Griffith University, Gold Coast Campus, Queensland, Australia

Zhen Lei Center for Biometrics and Security Research \& National Laboratory of Pattern Recognition, Institute of Automation, Chinese Academy of Sciences, Beijing, China

Lu Leng School of Electrical and Electronic Engineering, Yonsei University, Seoul, South Korea

Key Laboratory of Non-destructive Test (Ministry of Education), Nanchang Hangkong University, Nanchang, P.R. China

Jianjie Li Institute of Automation, Chinese Academy of Sciences, Beijing, People's Republic of China

Peng Li Institute of Automation, Chinese Academy of Sciences, Beijing, People's Republic of China

Shengzhe Li Inha University, Incheon, Korea

Stan Z. Li Center for Biometrics and Security Research \& National Laboratory of Pattern Recognition, Institute of Automation, Chinese Academy of Sciences, Beijing, China

Yung-hui Li Language Technology Institute, Carnegie Mellon University, Pittsburgh, PA, USA

Meng-Hui Lim Department of Computer Science, Hong Kong Baptist University, Kowloon Tong, Hong Kong

Zhouchen Lin Peking University, Beijing, China

James J. Little University of British Columbia, Vancouver, BC, Canada

Laura L. Liu Biometrics Research Centre, The Hong Kong Polytechnic University, Hung Hom, Hong Kong

Zongyi Liu Personal device, Device and Services, Microsoft Corporation, Redmond, WA, USA

Judith Liu-Jimenez GUTI (University Group for Identification Technologies), University Carlos III of Madrid, Leganes, Madrid, Spain

Guangming Lu School of Computer Science and Technology, Shenzhen Graduate School, Harbin Institute of Technology, Shenzhen, China 
Marcel Lüthi Computer Science Department, University of Basel, Basel, Switzerland

Niels Lynnerup Laboratory of Biological Anthropology, Institute of Forensic Medicine, University of Copenhagen, Copenhagen, Denmark

Jean-François Mainguet CEA, LETI, MINATEC Campus, University of Grenoble Alpes, Grenoble, France

Emanuele Maiorana Section of Applied Electronics, Department of Engineering, Roma Tre University, Rome, Italy

Sotiris Malassiotis Informatics and Telematics Institute, Center for Research and Technology Hellas, Thessaloniki, Greece

Davide Maltoni Department of Computer Science and Engineering, University of Bologna, Bologna, Cesena, Italy

Kevin Mangold National Institute of Standards and Technology, Gaithersberg, MD, USA

Sébastien Marcel Idiap Research Institute, Martigny, Switzerland

Gian Luca Marcialis Department of Electrical and Electronic Engineering, University of Cagliari, Cagliari, Italy

Judith Markowitz J. Markowitz, Consultants, Chicago, IL, USA

Alvin F. Martin National Institute of Standards and Technology, Gaithersburg, MD, USA

Aleix M. Martinez Department of Electrical and Computer Engineering, Ohio State University, Columbus, OH, USA

Marcos Martinez-Diaz Biometric Recognition Group - ATVS, Escuela Politecnica Superior, Universidad Autonoma de Madrid, Campus de Cantoblanco, Madrid, Spain

John S.D. Mason Swansea University, Swansea, UK

James R. Matey Electrical and Computer Engineering Department, US Naval Academy, Annapolis, MD, USA

Driss Matrouf CERI, University of Avignon, Avignon, France

Takashi Matsumoto Waseda University, Tokyo, Japan

Rene McIver SecureKey Technologies Inc., Toronto, ON, Canada

Gerard Medioni University of Southern California, Los Angeles, CA, USA

Didier Meuwly Netherlands Forensic Institute, The Hague, The Netherlands

Ross J. Micheals National Institute of Standards and Technology, Gaithersberg, MD, USA

Oscar Miguel-Hurtado School of Engineering and Digital Arts, University of Kent, Canterbury, Kent, UK 
Krystian Mikolajczyk School of Electronics and Physical Sciences, University of Surrey, Guildford, Surrey, UK

Philippos Mordohai Stevens Institute of Technology, PA, USA

Ken Moses Forensic Indentification Services, San Franscisco, CA, USA

Crystal Muang Department of Computer Science, Rutgers University, Piscataway, NJ, USA

Robert Mueller Next Biometrics AS, Next Biometrics, Nesoya, Norway

Tanvir Singh Mundra Biometrics Research Laboratory, Department of Electrical Engineering, Indian Institute of Technology Delhi, New Delhi, India

Taihei Munemoto Carnegie Mellon University, Pittsburgh, PA, USA

Daigo Muramatsu Department of Intelligent Media, The Institute of Scientific and Industrial Research, Osaka University, Osaka, Japan

Manabu Nakano Information-Technology Promotion Agency (IPA), Bunkyo-ku, Tokyo, Japan

Karthik Nandakumar Institute for Infocomm Research, A*STAR, Fusionopolis, Singapore

Ramkumar Narayanswamy Omni Vision CDM Optics, Boulder, CO, USA

Alessandro Neri Section of Applied Electronics, Department of Engineering, Roma Tre University, Rome, Italy

Jie Ni Department of Electrical \& Computer Engineering, Center for Automation Research, UMIACS, University of Maryland, College Park, MD, USA

Matthias Niesing Secunet Security Networks AG, Essen, Germany

Mark Nixon School of Electronics and Computer Science, University of Southampton, Southampton, UK

Afzel Noore West Virginia University, Morgantown, WV, USA

Kevin O'Connor Purdue University, West Lafayette, IN, USA

Javier Ortega-Garcia ATVS - Biometric Recognition Group, Escuela Politecnica Superior, Universidad Autonoma de Madrid, Madrid, Spain

Lisa Osadciw Syracuse University, Syracuse, NY, USA

Asem Othman Michigan State University, East Lansing, MI, USA

Chen Tai Pang Institute for Infocomm Research, A*STAR, Singapore

Maja Pantic Department of Computing, Imperial College London, London, UK

Sung W. Park Carnegie Mellon University, Pittsburgh, PA, USA 
Geppy Parziale INVASIVECODE INC., San Francisco, CA, USA

Georgios Passalis Department of Computer Science, ECE and Biomedical Engineering, University of Houston, Houston, TX, USA

Vishal M. Patel Center for Automation Research, UMIACS, University of Maryland, College Park, MD, USA

Ioannis Patras Queen Mary, University of London, London, UK

V. Paúl Pauca Wake Forest University, Winston-Salem, NA, USA

Nikola Pavešić Faculty of Electrical Engineering, University of Ljubljana, Ljubljana, Slovenia

Maria Pavlou University of Sheffield, Mappin Street, Sheffield, UK

Xiaoming Peng School of Automation Engineering, University of Electronic Science and Technology of China, Chengdu, Sichuan, China

School of Computer Science and Software Engineering, The University of Western Australia, Crawley, Perth, WA, Australia

Takis Perakis Department of Computer Science, ECE and Biomedical Engineering, University of Houston, Houston, TX, USA

Matti Pietikäinen Machine Vision Group, Department of Electrical and Information Engineering, University of Oulu, Oulu, Finland

Fernando Podio Computer Security Division, National Institute of Standards and Technology, Gaithersburg, MD, USA

Norman Poh Department of Computing, University of Surrey, Guildford, Surrey, UK

Frank E. Pollick Department of Psychology, University of Glasgow, Glasgow, UK

Fred Preston British Standards Institute, London, UK

Ryan Rakvic Applied Physics Laboratory, The John Hopkins University, Annapolis, MD, USA

Narayanan Ramanathan University of Maryland, College Park, MD, USA

Daniel Ramos ATVS - Biometric Recognition Group, Escuela Politecnica Superior, Universidad Autonoma de Madrid, Madrid, Spain

Marek Rejman-Greene Home Office Scientific Development Branch, Sandridge, St Albans, Herts, UK

Douglas Reynolds Lincoln Laboratory, MIT, Lexington, MA, USA

Karl Ricanek University of North Carolina Wilmington, Wilmington, NC, USA

Samuel Rivera The Ohio State University, Columbus, OH, USA 
Daria La Rocca Section of Applied Electronics, Department of Engineering, Roma Tre University, Rome, Italy

Rubén Vera Rodriguez Swansea University, Swansea, UK

Fabio Roli Department of Electrical and Electronic Engineering, University of Cagliari, Cagliari, Piazza d'Armi, Italy

Sami Romdhani University of Basel, Basel, Switzerland

Arun Ross Michigan State University, East Lansing, MI, USA

Besma Roui-Abidi The University of Tennessee, Knoxville, TN, USA

Amit K. Roy-Chowdhury Department of Electrical Engineering, University of California, Riverside, CA, USA

Anthony P. Russo Atrua Technologies Inc., Campbell, CA, USA

Raul Sanchez-Reillo GUTI (University Group for Identification Technologies), University Carlos III of Madrid, Leganes, Madrid, Spain

Enkhbayar Sanduijav Hitachi Solutions, Ltd, London, UK

Aswin C. Sankaranarayanan University of Maryland, College Park, MD, USA

Sudeep Sarkar Computer Science and Engineering, University of South Florida, Tampa, FL, USA

Marios Savvides CMU CyLab Biometrics Center, Pittsburgh, PA, USA

Natalia A. Schmid Department of Computer Science and Electrical Engineering, West Virginia University, Morgantown, WV, USA

Adee A. Schoon Animal Behavior Group, Leiden University, Leiden, The Netherlands

Juergen Schroeter AT\&T Labs Research, Florham Park, NJ, USA

Michael E. Schuckers St. Lawrence University, Canton, NY, USA

Stephanie A.C. Schuckers Clarkson University, Potsdam, NY, USA

Dale Setlak AuthenTec, Inc., Melbourne, FL, USA

Yoichi Seto Advanced Institute of Industrial Technology, Tokyo Metropolitan University, Tokyo, Japan

Shiguang Shan Institute of Computing Technology, Chinese Academy of Sciences, Beijing, China

Vinay Sharma Ohio State University, Columbus, OH, USA

Dmytro Shevtsov KP VTI PJSC, Kyiv, Ukraine

Takashi Shinzaki Social Innovation Laboratories, Fujitsu Laboratories Ltd., Kawasaki, Japan 
Paulo E.X. Silveira Omni Vision CDM Optics, Boulder, CO, USA

Terence M. Sim School of Computing, National University of Singapore, Singapore, Singapore

Richa Singh Image Analysis and Biometrics Lab, IIIT-Delhi, New Delhi, India

Kate Smith-Miles Deakin University, Melbourne, VIC, Australia

Jung Soh University of Calgary, Calgary, AB, Canada

Colin Soutar Cyber Risk Services, Deloitte and Touche LLP, Arlington, VA, USA

Philip Statham Biometrics Consultant, Specialising in Standards and Security Cheltenham, Gloucestershire, UK

Alex Stoianov Office of the Information and Privacy Commissioner, Toronto, ON, Canada

Vitomir Štruc Faculty of Electrical Engineering, University of Ljubljana, Ljubljana, Slovenia

Ravichandran Subramanian Computer Science and Engineering, University of South Florida, Tampa, FL, USA

Yunlian Sun Computer Vision Laboratory, Department of Sciences and Information Technology, University of Sassari, Sassari, Italy

Jinli Suo Department of Automation, Tsinghua University, Beijing, China

Matthew Swayze Daon, Inc., Reston, VA, USA

Zahid Syed Lane Department of Computer Science and Electrical Engineering, West Virginia University, Morgantown, WV, USA

Elham Tabassi National Institute of Standards and Technology, Gaithersburg, MD, USA

Samir Tamer Ingersoll Rand Recognition Systems, Campbell, CA, USA

Xunqiang Tao Institute of Automation, Chinese Academy of Sciences, Beijing, People's Republic of China

Chin-Hung Teng Yuan Ze University, Taiwan, Republic of China

Andrew B.J. Teoh School of Electrical and Electronic Engineering, Yonsei University, Seoul, South Korea

Theoharis Theoharis Department of Computer Science, ECE and Biomedical Engineering, University of Houston, Houston, TX, USA

Michael Thieme International Biometric Group, A Novetta Solutions Company, New York, NY, USA 
Jason Thornton Department of Electrical and Computer Engineering, Carnegie Mellon University, Pittsburgh, PA, USA

Jie Tian Center for Biometrics and Security Research \& The Key Laboratory of Complex System and Intelligence Science Chinese Academy of Sciences, Institute of Automation Zhingguancun Donglu, Beijing, China

Catherine J. Tilton Standards \& Technology, Daon, Reston, VA, USA

Massimo Tistarelli Computer Vision Laboratory, Department of Sciences and Information Technology, University of Sassari, Sassari, Italy

George Toderici Department of Computer Science, ECE and Biomedical Engineering, University of Houston, Houston, TX, USA

Doroteo T. Toledano ATVS - Biometric Recognition Group, Escuela Politecnica Superior, Universidad Autonoma de Madrid, Madrid, Spain

Ted Tomonaga Konica Minolta Technology Center, Inc., Tokyo, Japan

Todd C. Torgersen Wake Forest University, Winston-Salem, NA, USA

Yasunari Tosa Retica Systems, Inc., Waltham, MA, USA

Anna Bori Toth Cyberisk Limited, London, UK

Alessandro Triglia OSS Nokalva, Inc., Somerset, NJ, USA

Ryan Triplett Biometric Services International, LLC, A National Biometric Security Project Company, Morgantown, WV, USA

Tinne Tuytelaars Department of Electrical Engineering, Katholieke Universiteit Leuven, Leuven, Belgium

Ambrish Tyagi Ohio State University, Columbus, OH, USA

Kaoru Uchida NEC Corporation, Kawasaki, Japan

David Usher Retica Systems, Inc., Waltham, MA, USA

Mayank Vasta Image Analysis and Biometrics Lab, IIIT-Delhi, New Delhi, India

Kalyan Veeramachaneni Syracuse University, Syracuse, NY, USA

Ashok Veeraraghavan University of Maryland, College Park, MD, USA

Thomas Vetter Computer Science Department, University of Basel, Basel, Switzerland

Peter Waggett Emerging Technology, IBM United Kingdom Ltd, Portsmouth, Hampshire, UK

Michael Wagner College of Engineering and Computer Science, Australian National University, Canberra, ACT, Australia

National Centre for Biometric Studies Pty Ltd, Canberra, Australia

Faculty of ESTeM, University of Canberra, Canberra, Australia 
Liang Wan City University of Hong Kong, Hong Kong, China

James L. Wayman College of Engineering, San Jose State University, San Jose, CA, USA

Brad Wing National Institute of Standards and Technology (NIST), Gaithersburg, MD, USA

Andreas Wolf Bundesdruckerei GmbH, Berlin, Germany

Lior Wolf The Blavatnik School of Computer Science, Tel-Aviv University, Tel Aviv, Israel

Damon L. Woodard Clemson University, Clemson, SC, USA

Zhizheng Wu Nanyang Technological University (NTU), Nanyang, Singapore

Xudong Xie Department of Automation, Tsinghua University, Beijing, China

Yilei Xu Microsoft, USA

Dylan Yaga Computer Security Division, Systems \& Emerging Technologies Security Research, National Institute of Standards and Technology, Gaithersburg, MD, USA

Chew-Yean Yam University of Southampton, Southampton, UK

Asahiko Yamada IT Research and Development Center, Toshiba Solutions Corporation, Tokyo, Japan

Junichi Yamagishi National Institute of Informatics, Chiyoda, Tokyo, Japan University of Edinburgh, Edinburgh, UK

Brian A. Yamashita Forensic Identification Operations Support Services, National Services and Research, Royal Canadian Mounted Police, Ottawa, ON, Canada

Ming-Hsuan Yang University of California, Merced, CA, USA

Weilong Yang Center for Biometrics and Security Research \& National Laboratory of Pattern Recognition, Institute of Automation, Chinese Academy of Sciences, Beijing, China

Bian Yang Gjøvik University College, Gjøvik, Norway

Jian Yang School of Computer Science and Technology, Nanjing University of Science and Technology, Nanjing, People's Republic of China

Jingyu Yang School of Computer Science and Technology, Nanjing University of Science and Technology, Nanjing, People's Republic of China

Xin Yang Institute of Automation, Chinese Academy of Sciences, Beijing, People's Republic of China

Wei-Yun Yau Institute for Infocomm Research, Singapore 
Dong Yi Center for Biometrics and Security Research \& National Laboratory of Pattern Recognition, Institute of Automation, Chinese Academy of Sciences, Beijing, China

Lin Yih Digital Applied Research and Technology Pte Ltd, Singapore, Singapore

Isao Yoshimura Tokyo University of Science, Shinjuku-ku, Tokyo, Japan

Mitsu Yoshimura Ritsumeikan University, Sakyo-ku, Kyoto, Japan

Pong C. Yuen Department of Computer Science, Hong Kong Baptist University, Kowloon Tong, Kowloon, Hong Kong

Yau Wei Yun Institute for Infocomm Research, A*STAR, Singapore

Arie Zeelenberg National Police Agency, Zoetermeer, The Netherlands

Gregory Zektser Booz Allen Hamilton, Inc., Herndon, VA, USA

Jasenko Zivanov University of Basel, Basel, Switzerland

David Zhang Biometrics Research Centre, Department of Computing, The Hong Kong Polytechnic University, Hung Hom, Kowloon, Hong Kong

Yangyang Zhang Center for Biometrics and Security Research \& The Key Laboratory of Complex System and Intelligence Science Chinese Academy of Sciences, Institute of Automation Zhingguancun Donglu, Beijing, China

Wei-Shi Zheng School of Information Science and Technology, Sun Yat-sen University, Guangzhou, Guangdong, People's Republic of China

Mingquan Zhou Beijing Normal University, Beijing, China

S. Kevin Zhou Siemens Corporate Research, Princeton, NJ, USA

Sujing Zhou Institute of Automation, Chinese Academy of Sciences, Beijing, People's Republic of China

Zhi-Hua Zhou National Key Lab for Novel Software Technology, Nanjing University, Nanjing, China

Song-Chun Zhu Department of Computer Science, University of California, Los Angeles, CA, USA

Xiangxin Zhu Center for Biometrics and Security Research \& National Laboratory of Pattern Recognition, Institute of Automation, Chinese Academy of Sciences, Beijing, China 